Time-dependent solutions for particle size segregation in shallow granular avalanches

Gray, JMNT and Shearer, M and Thornton, AR 2006

Manchester Institute for Mathematical Sciences

School of Mathematics

The University of Manchester

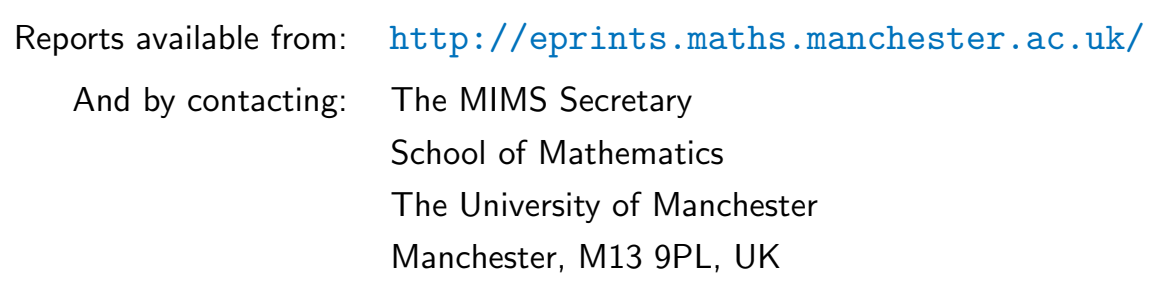




\title{
Time-dependent solutions for particle-size segregation in shallow granular avalanches
}

\author{
By J.M.N.T. Gray ${ }^{1}$, M. SheareR ${ }^{2} \&$ A.R. Thornton ${ }^{1}$ \\ ${ }^{1}$ School of Mathematics, University of Manchester, Manchester UK. \\ ${ }^{2}$ Department of Mathematics $\&$ Center for Research in Scientific Computation, \\ North Carolina State University, Raleigh, NC, USA.
}

\begin{abstract}
Rapid shallow granular free-surface flows develop in a wide range of industrial and geophysical flows, ranging from rotating kilns and blenders to rock-falls, snow slab-avalanches and debris-flows. Within these flows, grains of different sizes often separate out into inversely graded layers, with the large particles on top of the fines, by a process called kinetic sieving. In this paper, a recent theory is used to construct exact time-dependent two-dimensional solutions for the development of the particle-size distribution in inclined chute flows. The first problem assumes the flow is initially homogeneously mixed and is fed at the inflow with homogeneous material of the same concentration. Concentration shocks develop during the flow and the particles eventually separate out into inversely-graded layers sufficiently far downstream. Sections with a monotonically decreasing shock height, between these layers, steepen and break in finite time. The second problem assumes that the material is normally graded, with the small particles on top of the coarse ones. In this case, shock waves, concentration expansions, non-centred expanding shock regions and breaking shocks develop. As the parameters are varied, non-linearity leads to fundamental topological changes in the solution, and, in simple-shear, a logarithmic singularity prevents a steady-state solution from being attained.
\end{abstract}

Keywords: Particle-size segregation, kinetic sieving, inverse-grading, shocks.

\section{Introduction}

Particle-size segregation is notorious in bulk solids handling. Sometimes it can be used to our advantage, such as in mineral separation technology (Wills 1979), but often it presents a major technical hurdle in manufacturing processes, where grains need to be mixed together, or transported to another location. The scale of granular materials processing is vast, with applications in the bulk chemical, pharmaceutical, mining, food and agricultural industries. In many of these processes, such as in rotational mixers (Shinbrot \& Muzzio 1999) and huge continuous feed rotational kilns (Davidson et al. 2000), segregation occurs in shallow granular avalanches that develop in the free-surface layer of the flow. A knowledge of the segregation within these avalanches is therefore vital to understanding the segregation and mixing in more complex granular flows with solid-liquid phase transitions (Williams 1968, Gray \& Hutter 1997).

There are a number of mechanisms that drive particle-size segregation, but the dominant one in shallow granular free-surface flows is that of kinetic sieving 
(Bridgwater 1976), with the next most important effects being turbulent remixing, particle-density differences and grain-inertia effects at size ratios greater than ten (Thomas 2000). The basic kinetic sieving mechanism is simple. As the grains avalanche downslope there are fluctuations in the void space between the particles. When a void opens up under a layer of grains, the small particles are more likely to fall into the gap, because they are more likely to fit into the available space. The fines, therefore, percolate to the bottom of the flow and mass conservation dictates that there is a corresponding reverse flow of large particles towards the free surface. Kinetic sieving is so efficient in dry granular flows that in small scale experiments a layer of $100 \%$ coarse grains develops on top of a layer of $100 \%$ fines with a sharp concentration jump between them (Savage \& Lun 1988, Vallance \& Savage 2000). In geology this type of particle-size distribution is termed inverse-grading (Middleton \& Hampton 1976). The local size distribution can also have a subtle feedback onto the bulk flow. For instance, when the large particles are rougher than the small ones and there is strong shear through the depth of the avalanche, the large particles tend to congregate at the front and resist the motion. This leads to an instability in which the large particles are pushed to the sides to form stationary lateral levees (Pouliquen et al. 1997) that channel the flow and lead to significantly longer debris-flow run-out distances (Vallance 2000, Iverson \& Vallance 2001).

Despite the critical importance of segregation in both industrial and geophysical granular flows, there has been very little theoretical progress. Savage \& Lun (1988) were the first to derive a steady-state model using statistical mechanics and information entropy ideas, but it was overlooked for many years because of its apparent complexity. It also had the disadvantage that the percolation fluxes were independent of gravity, even though gravity is the fundamental driving mechanism for the kinetic sieving process. Gray \& Thornton (2005) used mixture theory to formulate mass and momentum balances for the large and small particles, which provided a natural way of introducing gravity into the theory. Assuming a linear velocity drag between the particles and that the fines carry less of the overburden pressure as they fall down through the gaps, Gray and Thornton derived an expression for the segregation flux in terms of the volume fraction of small particles. The resulting time-dependent segregation equation is considerably simpler and more general than that of Savage \& Lun (1988), but is still able to quantitatively reproduce the concentration jumps observed in the laboratory experiments of Savage \& Lun (1988) and Vallance \& Savage (2000). This is no coincidence, since the underlying mathematical structure of the two theories is the same in steady-state, even though the derivation and the interpretation of the coefficients are quite different. Gray \& Thornton's (2005) model represents the simplest possible theory for modelling three-dimensional time-dependent segregation, and its basic structure is inherent in more complex models that incorporate a dense-fluid in the pore space (Thornton, Gray \& Hogg 2005) and diffusive-remixing (Dolgunin \& Ulokov 1995, Gray \& Chugunov 2005).

In this paper, two exact solutions of the Gray and Thornton segregation equation are constructed; these yield detailed insight into the formation and evolution of some key segregation features, such as shock waves, expansion fans and inversely graded layers, that cannot be deduced easily from numerical simulations alone. One virtue of the two exact solutions is that they are a rigorous check that numerical solutions of the PDE capture accurately details of the segregation as it evolves. 


\section{The segregation equations and the bulk flow}

Gray \& Thornton's (2005) theory introduces a volume fraction $\phi$ of small particles per unit mixture volume that lies in the range

$$
0 \leq \phi \leq 1
$$

with $\phi=1$ corresponding to $100 \%$ small particles and $\phi=0$ corresponding to $100 \%$ coarse particles. The large particles are of the same density and occupy a volume fraction $1-\phi$. This simple approach implicitly assumes that the interstitial pore space is incorporated into the bulk density of the large and small particles. A more complex theory, which accounts for the buoyancy effects of an interstitial fluid of a different density to the particles (Thornton, Gray \& Hogg, 2005) leads to a similar formulation. In this section, we outline the derivation of the segregation equation, referring to Gray \& Thornton (2005) for the details.

Within the avalanche the bulk pressure $p$ is assumed to be hydrostatic through the depth $h$, which is measured normal to the chute, i.e.

$$
p=\rho g(h-z) \cos \zeta,
$$

where $\rho$ is the bulk density, $g$ is the constant of gravitational acceleration and $\zeta$ is the inclination angle of the chute. The bulk flow is assumed to be incompressible

$$
\frac{\partial u}{\partial x}+\frac{\partial v}{\partial y}+\frac{\partial w}{\partial z}=0
$$

where $x, y, z$ are coordinates in the downslope, cross-slope and normal directions to the chute, with unit coordinate vectors $\boldsymbol{i}, \boldsymbol{j}, \boldsymbol{k}$, respectively, and velocity $\boldsymbol{u}=$ $u \boldsymbol{i}+v \boldsymbol{j}+w \boldsymbol{k}$. In shallow granular free-surface flow models the bulk depth-averaged downslope and cross-slope velocities $(\bar{u}, \bar{v})$ are computed as a function of space and time, using prescribed velocity profiles. These profiles introduce shape factors into the momentum transport terms, which are equal to unity for plug flow and $4 / 3$ for simple shear. The bulk three-dimensional velocity field $\boldsymbol{u}(\boldsymbol{x}, t)$ can be reconstructed from $(\bar{u}, \bar{v})$ with a knowledge of the shape factors together with the incompressibility relation and the no normal velocity condition at the base to determine the bulk normal velocity

$$
w=-\int_{0}^{z} \frac{\partial u}{\partial x}+\frac{\partial v}{\partial y} d \xi
$$

Assumptions (2.2) and (2.3) are common to almost all models for granular freesurface flows from the hydraulic-type avalanches theories (e.g. Grigorian et al. 1967, Eglit 1983, Gray et al. 2003), to the dry Mohr-Coulomb (e.g. Savage \& Hutter 1989, Gray et al. 1999) and water saturated debris-flow models (Iverson 1997). All of these theories can be used to compute the bulk velocity field, but, for simplicity it is prescribed in this paper.

The key element of Gray \& Thornton's (2005) segregation theory is the use of the normal component of the constituent momentum balances to derive two equations that govern the percolation of the large and small particles. In non-dimensional variables these equations are

$$
\begin{aligned}
w^{l} & =w+S_{r} \phi, \\
w^{s} & =w+S_{r}(1-\phi),
\end{aligned}
$$


where $w^{l}$ and $w^{s}$ are the normal percolation velocities of the large and small particles, respectively. The non-dimensional segregation number $S_{r}$ is defined as

$$
S_{r}=\frac{L B g \cos \zeta}{c H U},
$$

where $c$ is an inter-particle drag coefficient, the non-dimensional factor $B$ determines the magnitude of the pressure perturbations that drive the flow and the avalanche has thickness $H$, length $L$ and downslope velocity magnitude $U$. In general the nondimensional segregation number $S_{r}$ may depend on a number of additional factors such as the local shear, the particle size difference and the particle roughness. In this paper $S_{r}$ is assumed to be a constant that can be calibrated from experiment.

The large particle percolation equation (2.5) simply implies that the large particles move upwards, relative to the bulk, until they separate out into a pure coarse phase. Equation (2.6) similarly expresses the property that small particles filter down until they separate out. Substituting the small particle percolation velocity (2.6) into the small particle mass balance equation

$$
\frac{\partial \phi}{\partial t}+\frac{\partial}{\partial x}\left(\phi u^{s}\right)+\frac{\partial}{\partial y}\left(\phi v^{s}\right)+\frac{\partial}{\partial z}\left(\phi w^{s}\right)=0,
$$

together with the assumption that the downslope and cross-slope velocity components of the small particles are the same as those of the bulk flow

$$
u^{s}=u, \quad v^{s}=v,
$$

yields a non-dimensional segregation equation for the volume fraction of small particles

$$
\frac{\partial \phi}{\partial t}+\frac{\partial}{\partial x}(\phi u)+\frac{\partial}{\partial y}(\phi v)+\frac{\partial}{\partial z}(\phi w)-\frac{\partial}{\partial z}\left(S_{r} \phi(1-\phi)\right)=0 .
$$

The first four terms together with the bulk incompressibility (2.3) simply express the fact the volume fraction is advected passively with the flow. Whilst the final term on the lefthand-side is responsible for the separation of the particles into inversely graded layers.

Gray \& Thornton (2005) and Thornton, Gray \& Hogg (2005) showed that sharp concentration jumps or shocks appear in steady-state flows. A generalized time-dependent jump condition across such a shock can easily be derived from the divergence structure of (2.10). Let $z=z(x, y, t)$ be a shock surface, with spacetime normal $\mathbf{N}=\left(z_{t}, z_{x}, z_{y},-1\right)$. Observe that equation equation (2.10) has the divergence form DivG $=0$, where Div is the space-time divergence and

$$
\mathbf{G}=\left(\phi, \phi u, \phi v, \phi w-S_{r} \phi(1-\phi)\right) .
$$

Consequently, the normal component of $\mathbf{G}$ is continuous across the shock: $\mathbf{N}$. $\llbracket \mathbf{G} \rrbracket=$ 0 , where the jump bracket $\llbracket f \rrbracket=f^{+}-f^{-}$denotes the jump in a piecewise continuous function $f(x, y, z, t)$ across the shock. Thus,

$$
z_{t} \llbracket \phi \rrbracket+z_{x} \llbracket \phi u \rrbracket+z_{y} \llbracket \phi v \rrbracket-\llbracket \phi w \rrbracket+\llbracket S_{r} \phi(1-\phi) \rrbracket=0 .
$$

Dividing by $\llbracket \phi \rrbracket$, and assuming that the velocity $(u, v, w)$ is continuous, we obtain the equation

$$
\frac{\partial z}{\partial t}+u \frac{\partial z}{\partial x}+v \frac{\partial z}{\partial y}-w=S_{r}\left(\phi^{+}+\phi^{-}-1\right) .
$$


In the exact solutions derived in this paper, each shock $z=z_{\gamma}(x, y, t)$ is given an integer subscript $\gamma=1,2,3, \ldots$ to uniquely identify it.

The upper and lower boundaries of the avalanche, the surface $z=s(x, y, t)$ and the bottom $z=b(x, y)$, are special cases of the jump conditions in which there is no normal flow of small particles across the boundary. It follows from (2.12) that the surface and basal boundary conditions are

$$
\begin{aligned}
\phi\left[\frac{\partial s}{\partial t}+u^{s} \frac{\partial s}{\partial x}+v^{s} \frac{\partial s}{\partial y}-w^{s}\right] & =S_{r} \phi(1-\phi), \quad \text { on } z=s \\
\phi\left[\frac{\partial b}{\partial t}+u^{b} \frac{\partial b}{\partial x}+v^{b} \frac{\partial b}{\partial y}-w^{b}\right] & =S_{r} \phi(1-\phi), \quad \text { on } \quad z=b .
\end{aligned}
$$

The square-bracketed term on the lefthand-side of these equations is zero, due to the kinematic boundary condition at the surface and base of the avalanche (e.g. Gray et al 1999; Gray et al 2003). The surface and basal boundary conditions therefore reduce to

$$
\phi(1-\phi)=0, \quad \text { on } \quad z=s, b,
$$

which is satisfied when either $\phi=0$ or $\phi=1$ at the surface and base.

\section{Steady-uniform flows}

In this paper we restrict attention to steady uniform flow, meaning granular flow in which the layer has uniform thickness, the bulk downslope velocity depends only on the depth variable $z$, and the cross-slope and normal velocity components are assumed to be zero. In these circumstances, the avalanche depth sets the length scale in $z$, so that the avalanche has unit depth in nondimensional variables and the velocity field is

$$
u=u(z), \quad v=0, \quad w=0, \quad \text { in } \quad 0 \leq z \leq 1
$$

The stretching transformation

$$
x=\tilde{x} / S_{r}, \quad t=\tilde{t} / S_{r}, \quad z=\tilde{z},
$$

can be used to rescale the segregation equation (2.10) into a convenient parameter independent form

$$
\frac{\partial \phi}{\partial t}+\frac{\partial}{\partial x}(\phi u)-\frac{\partial}{\partial z}(\phi(1-\phi))=0
$$

where the tildes are dropped for simplicity, and the associated jump condition is

$$
\frac{\partial z_{\gamma}}{\partial t}+u \frac{\partial z_{\gamma}}{\partial x}=\left(\phi^{+}+\phi^{-}-1\right)
$$

The transformation (3.2) implies that the grains take greater distances to segregate into inversely graded layers and take longer to do so for smaller $S_{r}$. All solutions are constructed in the stretched coordinate system and mapped back to the physical coordinate system using the transformation (3.2) if and when necessary. 


\section{Segregation in a homogeneous chute flow}

For our first problem we will consider the time-dependent version of Gray \& Thornton's (2005) steady-state problem in which a steady-uniform avalanche that is initially homogeneously mixed with concentration $\phi_{0}$ is continuously fed at $x=0$ with material of the same concentration $\phi_{0}$. The problem is therefore specified by the initial and boundary conditions

$$
\begin{array}{ccc}
\phi(x, z, 0)=\phi_{0}, & \text { in } \quad 0 \leq z \leq 1, & 0 \leq x<\infty, \\
\phi(0, z, t)=\phi_{0}, & \text { in } \quad 0 \leq z \leq 1, & 0 \leq t<\infty, \\
\phi(1-\phi)=0, & \text { on } \quad z=0,1, & 0 \leq t<\infty,
\end{array}
$$

where (4.3) is the no normal flux condition at the boundaries. Within the interior of the flow the small particles immediately start to percolate down towards the bottom of the avalanche and the large ones are pushed towards the free-surface. Except near boundaries the characteristics imply that the small particle concentration stays at $\phi_{0}$ and is swept downstream. At the lower boundary, however, there are simply no more large particles to be pushed up and the no normal flux condition (4.3) implies that the small particles separate out into a pure phase, creating a sharp concentration jump, or shock, with the initial mixture. Assuming that $\phi^{+}=\phi_{0}$ and $\phi^{-}=1$ on either side of the shock, (3.4) with $\gamma=1$ reduces to

$$
\frac{\partial z_{1}}{\partial t}+u\left(z_{1}\right) \frac{\partial z_{1}}{\partial x}=\phi_{0}
$$

The method of characteristics (e.g. Sneddon 1957) expresses differentiable solutions in the form $F(\lambda, \mu)=0$, where $F$ is an arbitrary function of the characteristic variables $\lambda(x, z, t)$ and $\mu(x, z, t)$, which are integrals of the characteristic equations

$$
\frac{d t}{1}=\frac{d x}{u\left(z_{1}\right)}=\frac{d z_{1}}{\phi_{0}} .
$$

We find expressions for $\lambda$ and $\mu$ by solving pairs of these equations. Integrating the first and third equations, we obtain

$$
z_{1}=\phi_{0} t+\lambda
$$

The second and third equations can be integrated for general velocity fields by introducing a depth-integrated velocity coordinate (Gray \& Thornton 2005)

$$
\psi=\int_{0}^{z} u\left(z^{\prime}\right) d z^{\prime}
$$

to give

$$
\psi_{1} \equiv \psi\left(z_{1}\right)=\phi_{0} x+\mu .
$$

(Here and elsewhere, we abbreviate functional dependences; thus, $\psi_{1}=\psi\left(z_{1}\right)$, and equation (4.8) may be regarded as relating $z_{1}$ to $x$ along a characteristic.) Note that the depth-integrated velocity coordinate lies in the range $0 \leq \psi \leq 1$ provided the downslope velocity is suitably scaled in the non-dimensionalisation process.

The full solution surface can be expressed as $\lambda=f(\mu)$, where $f$ is an arbitrary function. In this case the solution is particularly simple. Since (4.6) is independent 
of $x$ and the initial shock height $z_{1}(x, 0)=0$, it follows that the shock is horizontal and moves upwards linearly in time:

$$
z_{1}=\phi_{0} t
$$

for the material that was initially in the chute. For the material that enters the chute, the shock emanates from the origin, $\psi_{1}=0$, so (4.8) implies that the solution is independent of time

$$
\psi_{1}=\phi_{0} x
$$

The solution therefore consists of a time-dependent horizontal section that propagates upwards linearly in time and is swept downstream to reveal the steady-state solution adjacent to the inflow.

At the surface of the avalanche a similar jump develops. This time there are no more small particles available to percolate downwards and the large particles separate out to form a concentration shock with the bulk mixture. Assuming that $\phi^{+}=0$ and $\phi^{-}=\phi_{0}$ the jump condition (3.4) implies

$$
\frac{\partial z_{2}}{\partial t}+u \frac{\partial z_{2}}{\partial x}=-\left(1-\phi_{0}\right) .
$$

This can be solved in the same way as for the bottom jump. It consists of a horizontal part that propagates linearly downwards with time

$$
z_{2}=1-\left(1-\phi_{0}\right) t
$$

and is swept downstream to reveal the steady-state part

$$
\psi_{2}=1-\left(1-\phi_{0}\right) x .
$$

The concentration jump solutions (4.9), (4.10), (4.12) and (4.13) hold for any positive velocity field $u(z)$ with an isolated zero at the origin. Specific solutions are constructed in this paper for the linear velocity field

$$
u=\alpha+2(1-\alpha) z, \quad 0 \leq z \leq 1 .
$$

The parameter $\alpha$ allows the velocity to vary from plug-flow $(\alpha=1)$ to simple shear $(\alpha=0)$ and provides a good leading order approximation to more complex nonlinear velocity fields (e.g. Gray \& Thornton 2005). The corresponding depth integrated coordinate

$$
\psi=\alpha z+(1-\alpha) z^{2}
$$

can be inverted to give $z=z(\psi)$, i.e.

$$
z=\left\{\begin{array}{cc}
\psi, & \alpha=1, \\
\frac{-\alpha+\sqrt{\alpha^{2}+4(1-\alpha) \psi}}{2(1-\alpha)}, & \alpha \neq 1 .
\end{array}\right.
$$

The development of the upper and lower shocks is shown in Fig. 1 for a linear velocity field with $\alpha=0.1$. The position of the transition points between the steadystate and horizontal time-dependent sections of the lower and upper shocks are (respectively)

$$
\begin{aligned}
& x_{1}=\alpha t+(1-\alpha) \phi_{0} t^{2}, \\
& x_{2}=(2-\alpha) t-(1-\alpha)\left(1-\phi_{0}\right) t^{2},
\end{aligned}
$$




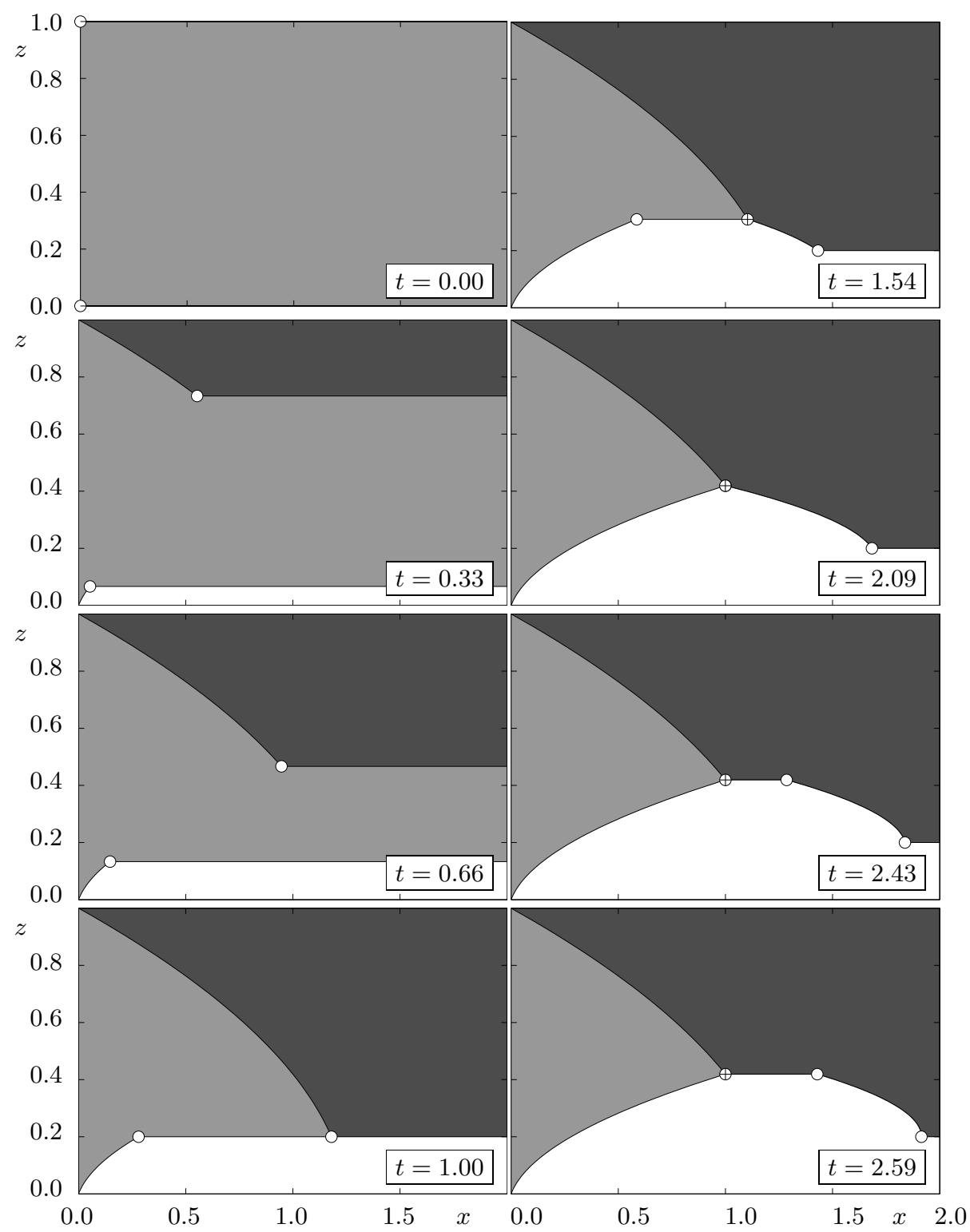

Figure 1. The evolution of the volume fraction of small particles $\phi$ as a function of $x$ and $z$. The particles enter the chute at $x=0$ and are swept downstream from left to right by a linear velocity field with a large shear component given by parameter $\alpha=0.1$. The white region corresponds to $100 \%$ small particles, the dark region to $100 \%$ large particles and the light grey region to the mixed region with concentration $\phi_{0}=0.2$. The circular markers correspond to the position of the transition points and the ' $\oplus$ ' is the triple-point.

and are shown by the open circular markers in the lefthand panels of Fig. 1. In plug-flow the two transitions propagate at the same speed, but when there is shear the upper transition $x_{2}$ moves faster than the lower transition $x_{1}$ in response to the larger velocities at the free-surface; thus, $x_{1}<x_{2}$ for $t>0$. 


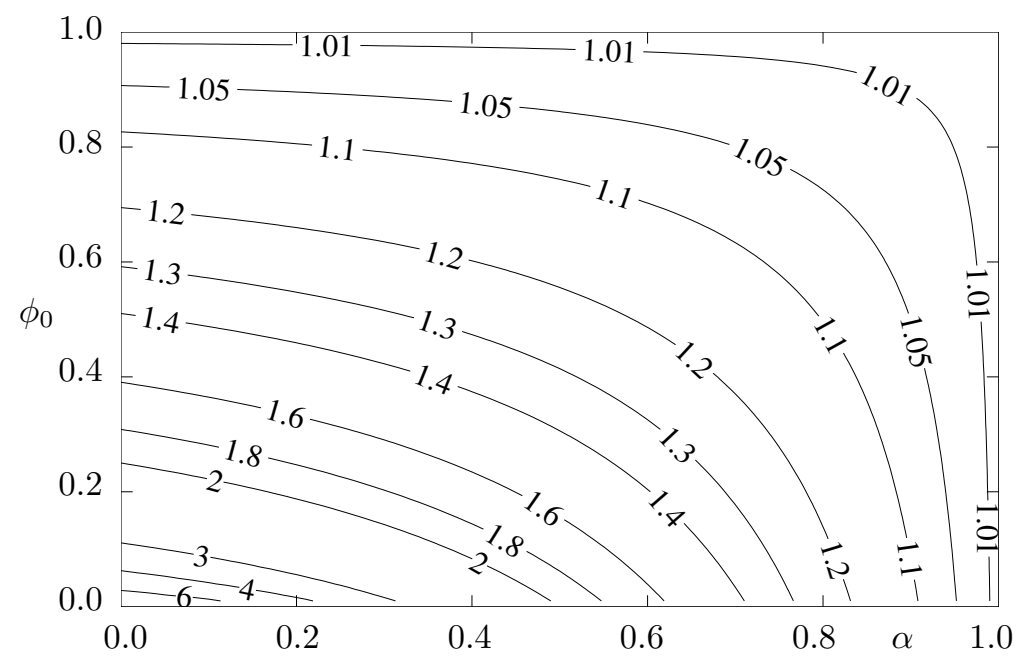

Figure 2. A contour plot of the time $t_{\text {steady }}$ for the two steady-state branches of the shock to meet at the steady-state triple-point as a function of velocity profile $\alpha$ and the inflow concentration $\phi_{0}$.

The upper and lower horizontal shocks meet where $z_{1}=z_{2}$. From (4.9), (4.12), this occurs at $t=1$, at height $z=\phi_{0}$, and over the range $x>x_{m}$, where $x_{m}$ is given by (4.18) at $t=1$ :

$$
x_{m}=1+(1-\alpha) \phi_{0} .
$$

For $t>1$ the resulting shock with $\phi^{+}=0$ and $\phi^{-}=1$ evolves according to the jump condition (3.4), which reduces to the scalar conservation law (resembling the inviscid Burgers equation)

$$
\frac{\partial z_{3}}{\partial t}+u \frac{\partial z_{3}}{\partial x}=0, \quad u=u\left(z_{3}\right)
$$

Given the initial position and time $\left(x_{0}, z_{0}, t_{0}\right)$ this has the simple solution

$$
z_{3}=z_{0}, \quad \text { on } \quad x=x_{0}+u\left(z_{0}\right)\left(t-t_{0}\right)
$$

It follows that when the upper and lower laterally uniform (horizontal) timedependent shocks meet, a third laterally uniform shock is formed that is stationary and advected downstream:

$$
z_{3}=\phi_{0}, \quad x>x_{m}+u\left(\phi_{0}\right)(t-1) .
$$

For plug flow $(\alpha=1)$, the solution is now at equilibrium, but for shear flow $\alpha<1$, there is subsequent dynamic behavior, so we now discuss only the case $\alpha<1$. In the region $x<x_{m}$ the upper and lower shocks do not intersect. Instead the lower horizontal shock (4.13) continues to propagate upwards intersecting the steady branch (4.8) of the top shock at a triple-point between the large, small and mixed regions:

$$
x_{\text {triple }}=\frac{1-\alpha \phi_{0} t-(1-\alpha) \phi_{0}^{2} t^{2}}{\left(1-\phi_{0}\right)}, \quad z_{\text {triple }}=\phi_{0} t .
$$




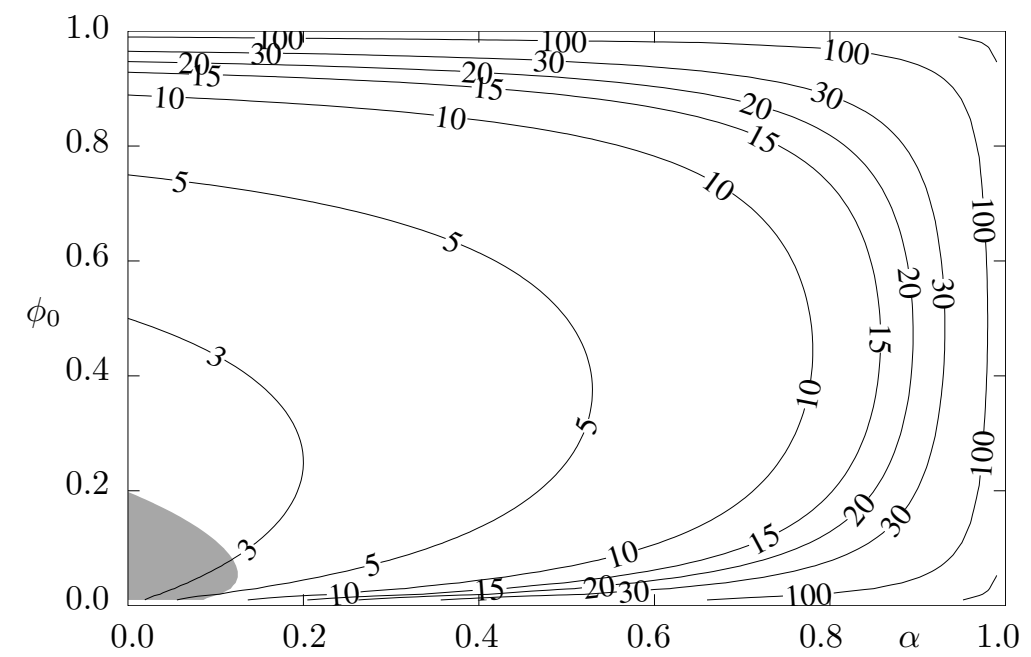

Figure 3. A contour plot of the time $t_{\text {break }}$ for the third shock to break as a function of velocity profile $\alpha$ and the inflow concentration $\phi_{0}$. The shaded region shows the region of parameter space where the shock breaks before the steady-state triple point forms.

This construction continues until the two steady-state branches meet at time

$$
t_{\text {steady }}=\frac{-\alpha+\sqrt{\alpha^{2}+4 \phi_{0}(1-\alpha)}}{2 \phi_{0}(1-\alpha)},
$$

at the steady-state triple-point position

$$
x_{\text {steady }}=1, \quad \psi_{\text {steady }}=\phi_{0} .
$$

The triple-point is illustrated in Fig. 1 using a ' $\oplus$ ' marker. It moves upstream from the initial point of meeting $x_{m}$ to its final steady-state position $x_{\text {steady }}$. The time it takes to reach this point is plotted in Fig. 2, which shows that it takes longer to develop with decreasing $\alpha$ and $\phi_{0}$.

The solution upstream of the steady triple-point has reached equilibrium, but downstream, the interface between the large and small particles continues to evolve. There are three distinct sections whose dynamics are all controlled by the conservation law equation (4.20). As can be seen from the general solution (4.21) the initial position and time $\left(x_{0}, z_{0}, t_{0}\right)$ at which the shock is generated are of crucial importance. It has already been shown that the shock is horizontal with height $\phi_{0}$ in a region propagating downstream. The remaining parts of the shock are governed by the upstream motion of the triple-point as it moves to its steady position. At a given height $z \in\left[z_{m}, z_{\text {triple }}\right]$ equations (4.23) imply that its initial position is

$$
x_{0}=\left[1-\alpha z-(1-\alpha) z^{2}\right] /\left(1-\phi_{0}\right),
$$

at time $t_{0}=z / \phi_{0}$. The dynamic part of the shock is therefore given by

$$
x=x_{0}+u(z)\left(t-t_{0}\right) .
$$

Once the triple-point reaches its steady-state position the initial conditions do not change and a straight horizontal shock of height

$$
z_{3}=z_{\text {steady }}, \quad \text { with } \quad x_{\text {steady }}<x<x_{\text {steady }}+u\left(z_{\text {steady }}\right)\left(t-t_{\text {steady }}\right),
$$


is formed. The development of the three sections of the third shock is shown in the righthand panels of Fig. 1. When there is shear, points that are higher up in the flow move faster downstream than those below them. As a result the central dynamic region of $z_{3}$ steepens and eventually breaks at $z=z_{m}$ (where the interface is steepest), at time

$$
t_{\text {break }}=\frac{\alpha+2(1-\alpha) \phi_{0}\left(2-\phi_{0}\right)}{2(1-\alpha) \phi_{0}\left(1-\phi_{0}\right)}
$$

This indicates that the breaking-time is singular when $\alpha=1$ and the third shock does not break, consistent with the exact time-dependent solutions for plug-flow constructed by Gray \& Thornton (2005). The breaking-time also tends to infinity as the initial concentration approaches either zero or unity. This is because the dynamic region of $z_{3}$ is confined to a very narrow lateral band in which the velocities become increasingly close to one another as $\phi_{0}$ approaches these limiting states and it therefore takes an increasingly long time to break. The full parameter dependence of the breaking-time on $\alpha$ and $\phi_{0}$ is illustrated in Fig. 3. This shows that there is a small (shaded) region of parameter space for high shear and low initial concentrations in which the third shock breaks prior to the steady triplepoint forming. Numerical solutions indicate that after the shock breaks a complex solution is formed that includes dynamic expansion fans and shocks, but a detailed analysis of this is left to a future paper.

\section{Segregation from a normally graded initial state}

The second problem also leads to shock wave-breaking and is the time-dependent version of Thornton, Gray \& Hogg's (2005) normally graded steady-state flows. Initially the avalanche is assumed to be normally graded, with a layer of small particles separated from a layer of large particles below by a concentration discontinuity along $z=z_{r}$. The avalanche is fed at $x=0$ with the same normally graded layered material and satisfies the no normal flux conditions at the surface and the base of the avalanche, i.e.

$$
\begin{aligned}
& \phi(x, z, 0)=\left\{\begin{array}{ll}
1, & z_{r} \leq z \leq 1, \\
0, & 0 \leq z<z_{r},
\end{array} \quad 0 \leq x<\infty\right. \\
& \phi(0, z, t)=\left\{\begin{array}{ll}
1, & z_{r} \leq z \leq 1, \\
0, & 0 \leq z<z_{r},
\end{array} \quad 0 \leq t<\infty\right. \\
& \phi(1-\phi)=0, \quad z=0,1, \quad 0 \leq x<\infty \quad 0 \leq t<\infty .
\end{aligned}
$$

Thornton, Gray \& Hogg (2005) have performed numerical simulations of this problem, which indicate that there is a region adjacent to the inflow that rapidly attains steady-state, a downstream region where the flow is time-dependent and laterally uniform and a transition region between the two, where there is complex spatial and temporal behaviour. The problem will therefore be solved in the steady-state and laterally uniform regions before the transition is considered. 


\section{(a) The steady problem}

Neglecting the time-derivatives in the segregation equation (3.3) and expanding the remaining terms yields a first order quasi-linear equation of the form

$$
u \frac{\partial \phi}{\partial x}+(2 \phi-1) \frac{\partial \phi}{\partial z}=0
$$

which may be solved by the method of characteristics. The small particle concentration $\phi$ is equal to a constant $\phi_{\lambda}$ along the characteristic curve given by the subsidiary equation

$$
\frac{d x}{u(z)}=\frac{d z}{2 \phi_{\lambda}-1}
$$

This may be integrated for general positive definite velocity fields with an isolated zero at the origin, by using the depth-integrated velocity coordinates defined in (4.7) to give

$$
\psi=\psi_{\lambda}+\left(2 \phi_{\lambda}-1\right)\left(x-x_{\lambda}\right),
$$

where $\left(x_{\lambda}, \psi_{\lambda}\right)$ is the starting position in mapped coordinates. Characteristic curves on which $\phi_{\lambda}>1 / 2$ propagate up through the avalanche, whilst curves with $\phi_{\lambda}<$ $1 / 2$ propagate down towards the base of the flow. It follows that at the inflow concentration discontinuity, which lies at $z=z_{r}$, or, $\psi=\psi_{r}$ in mapped coordinates, the characteristics diverge from one another and a centred expansion fan is formed with concentration

$$
\phi=\frac{1}{2}\left(1+\frac{\psi-\psi_{r}}{x}\right) \quad \text { in }\left|\psi-\psi_{r}\right|<x .
$$

The characteristic curve $\psi=\psi_{r}-x$ (on which $\phi=0$ ) lies along the lower boundary of the expansion fan and separates it from a pure region of large particles. It represents the trajectory of the first small particles percolating towards the base of the avalanche, which they reach at $x_{\text {bottom }}=\psi_{r}$. Similarly, the curve $\psi=\psi_{r}+x$ (on which $\phi=1$ ) separates the fan from a region of small particles above and represents the front of the first large particles that are being pushed up to the free-surface. They reach $z=1$ at $x_{t o p}=1-\psi_{r}$.

Once the small particles reach the base of the flow the no normal flux condition (5.3) implies that they separate out and a concentration shock is formed between the expansion fan and the pure phase. The steady-state version of the jump condition (3.4) with $\phi^{+}$given by $(5.7)$ and $\phi^{-}=1$ reduces to

$$
\frac{\partial \psi}{\partial x}=\frac{1}{2}\left(1+\frac{\psi-\psi_{r}}{x}\right),
$$

in mapped coordinates. This is a linear ODE that can easily be integrated subject to the initial condition that $\psi=0$ at $x=\psi_{r}$ to give the height of the bottom shock

$$
\psi_{1}=\psi_{r}+x-2 \sqrt{\psi_{r} x} .
$$

An analogous upper shock

$$
\psi_{2}=\psi_{r}-x+2 \sqrt{\left(1-\psi_{r}\right) x}
$$

Article submitted to Royal Society 
forms as the large particles separate out into a pure phase. The upper and lower shocks meet at

$$
x_{\text {steady }}=\left(\sqrt{\psi_{r}}+\sqrt{1-\psi_{r}}\right)^{2}, \quad \psi_{\text {steady }}=1-\psi_{r},
$$

and a third shock is formed between the inversely graded layers of large and small particles along the line

$$
\psi_{3}=\psi_{\text {steady }}, \quad \text { in } \quad x \geq x_{\text {steady }} .
$$

This solution has been investigated by Thornton, Gray \& Hogg (2005) for a range of linear and non-linear velocity profiles and values of the concentration discontinuity $z_{r}$. Readers are referred to this work for a detailed discussion of the steady solution.

\section{(b) The unsteady laterally uniform problem}

Sufficiently far downstream the lateral uniformity of the initial conditions implies that the solution is independent of the downslope coordinate $x$. In this case the expanded segregation equation (3.3) reduces to

$$
\frac{\partial \phi}{\partial t}+(2 \phi-1) \frac{\partial \phi}{\partial z}=0
$$

which, rather interestingly, is independent of the assumed velocity profile $u(z)$. Equation (5.13) has exactly the same form as the mapped steady-state problem (5.4) except that $x$ is replaced by $t$ and $\psi$ is replaced by $z$. The full solution may therefore be written down immediately. It consists of an expansion fan centred at $z=z_{r}$ within which the concentration is

$$
\phi=\frac{1}{2}\left(1+\frac{z-z_{r}}{t}\right)
$$

For small values of $t$, the expansion fan is confined to the interval $\left|z-z_{r}\right|<t$, the leading edges (where $\phi=0,1$ ) moving with speed $|2 \phi-1|=1$. After the leading edges hit the horizontal boundaries $z=0,1$, at times $t_{\text {bottom }}=z_{r}$ and $t_{\text {top }}=1-z_{r}$, the fan is contained in the interval $z_{1}(t)<z<z_{2}(t)$ bounded by the two shocks

$$
\begin{array}{ll}
z_{1}=z_{r}+t-2 \sqrt{z_{r} t}, & t_{\text {bottom }} \leq t \leq t_{3}, \\
z_{2}=z_{r}-t+2 \sqrt{\left(1-z_{r}\right) t}, & t_{\text {top }} \leq t \leq t_{3} .
\end{array}
$$

Finally, when the two shocks collide, at time $t_{3}=\left(\sqrt{z_{r}}+\sqrt{1-z_{r}}\right)^{2}$, the fan disappears and the solution is a single stationary shock

$$
z_{3}=1-z_{r}, \quad t \geq t_{3} .
$$

\section{(c) The full problem and the fan-interface}

Expanding the derivatives in the segregation equation (3.3) yields a first order quasi-linear equation

$$
\frac{\partial \phi}{\partial t}+u \frac{\partial \phi}{\partial x}+(2 \phi-1) \frac{\partial \phi}{\partial z}=0
$$




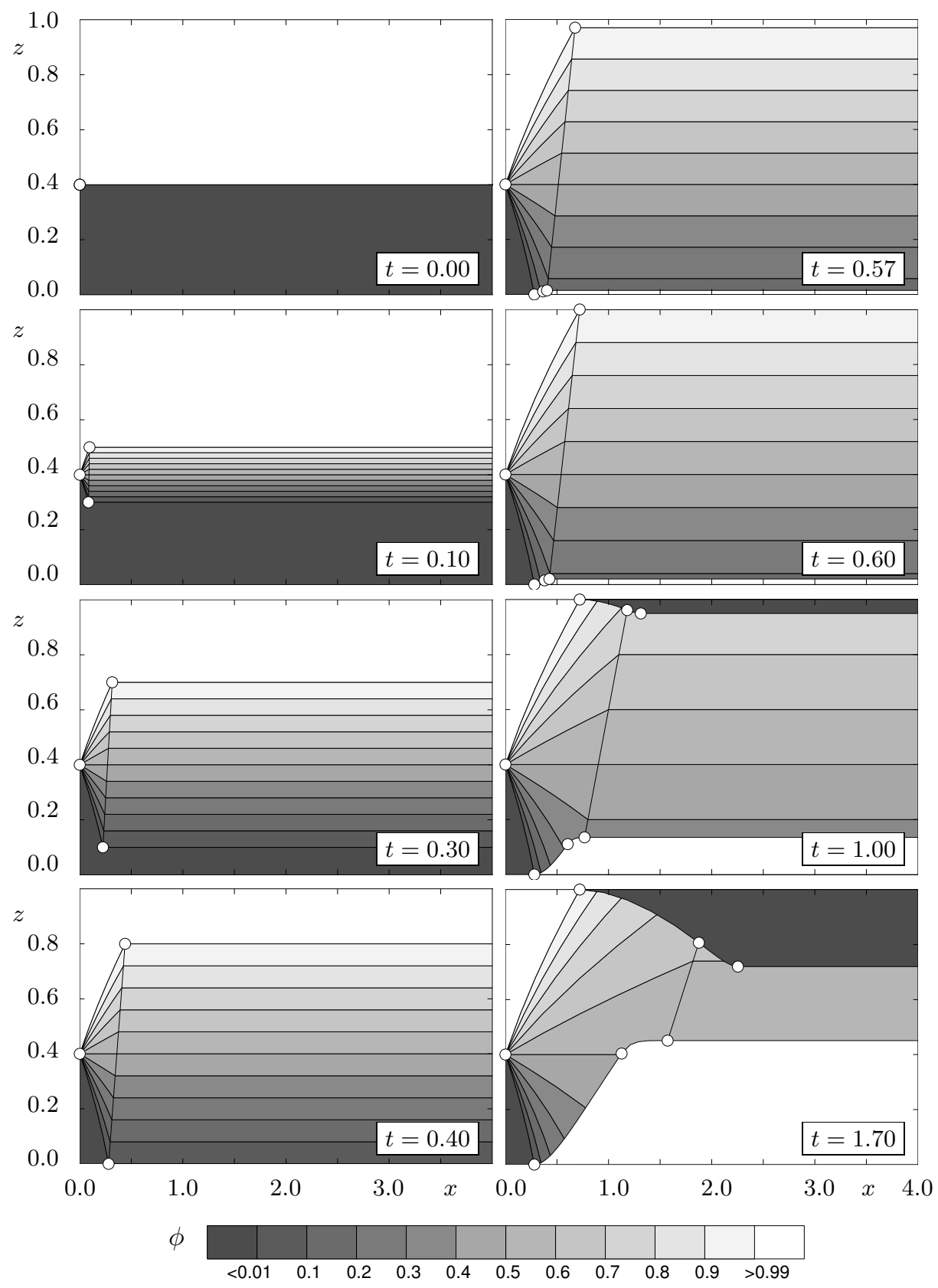

Figure 4. A series of contour plots showing the evolution of the volume fraction of small particles $\phi$ as a function of the downslope coordinate $x$ and avalanche depth $z$. The bulk linear shear flow is from left to right and is given by parameter $\alpha=1 / 2$. The normally graded particles enter the chute at $x=0$ and have a concentration discontinuity at $z_{r}=0.4$. The white circular markers correspond to the position of the transition points between steady, fully dynamic and unsteady laterally uniform behaviour and the marker with the plus sign is the triple-point. 
that governs the full problem. The method of characteristics implies that the concentration is equal to a constant $\phi_{\lambda}$ on characteristic curves (parameterized by $\lambda$ ) given by the subsidiary equations

$$
\frac{d t}{1}=\frac{d x}{u(z)}=\frac{d z}{2 \phi_{\lambda}-1} .
$$

The first and third can be integrated directly to give

$$
z=\left(2 \phi_{\lambda}-1\right) t+\lambda
$$

The first and second can then be integrated using (5.20) and the definition of the linear velocity field (4.14) to show that

$$
x=\alpha t+2(1-\alpha) \lambda t+(1-\alpha)\left(2 \phi_{\lambda}-1\right) t^{2}+f(\lambda)
$$

where $\lambda=z-\left(2 \phi_{0}-1\right) t$.

At $t=0$ the line $x=0$ separates the material that was initially in the chute from the material that subsequently enters the chute along $x=0$. Material initially in the chute is swept downstream, and experiences segregation in a time dependent expansion fan (5.14). On the other hand, material entering the chute is normally graded as it enters, and then begins to segregate in a steady expansion fan (5.7) centered at $x=0, z=z_{r}$. The interface between the two kinds of material points, those initially in the chute and those entering the chute, is a curve that we call the fan-interface; it is shown in the left panels of Fig.4. Points in the interface are located by tracking the characteristics (5.21) with initial location $x=0$. Thus, $f(\lambda) \equiv 0$. Within the time-dependent characteristic fan, $\phi_{\lambda}$ is given by (5.14), and the material on the time-dependent side of the fan-interface therefore lies along the straight line

$$
x=\left[\alpha+(1-\alpha)\left(z+z_{r}\right)\right] t,
$$

at time $t$. Equating the time-dependent and steady-state fan concentrations (5.14) and (5.7) also yields (5.22). This shows that the fan-interface (5.22) creates a continuous match between the steady-state and time-dependent fans. The solution for small times, therefore, consists of steady-state fan (5.7) upstream of the interface (5.22) and a time-dependent fan (5.14) downstream of it, which expand with increasing time. Above and below the fan are pure regions of small and large particles, as shown in the left-hand panels of Fig. 4. The fans continue to expand until the laterally uniform time-dependent sections intersect with the surface and base of the flow at times $t_{t o p}$ and $t_{\text {bottom }}$. Interaction with the no normal flux boundary condition (5.3) implies that shocks result from reflection at the boundary.

\section{(d) The fully dynamic bottom shock}

The subsequent flow involves horizontal shocks (discontinuities in $\phi$ ) propagating in from the surface and the base. The structure of the solutions in shown schematically in Fig.5. As indicated in the figure, the horizontal shocks do not match up neatly with the steady-state fan. As discussed earlier, the flow downstream consists of horizontal shocks, shown as sections CD (propagating upwards) and GH (propagating down) in Fig.5. Note that the structure of the solution propagating up from the base is significantly different from that of the waves descending 


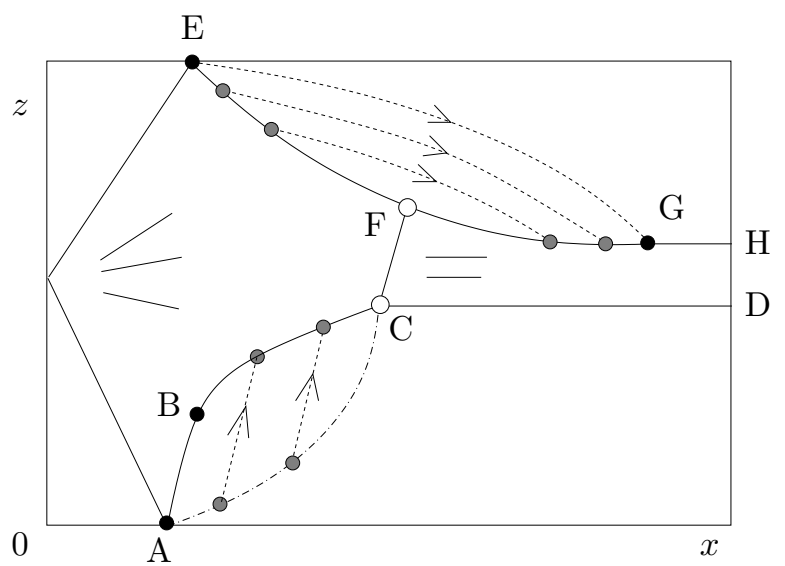

Figure 5. A sketch of the full solution once the upper and lower shocks have formed.

from the surface, due to the opposite velocity gradients encountered by the waves. In this subsection, we focus on the lower waves, the fully dynamic bottom shock.

The lower shock has three sections: the steady-state part AB (as in the steady state solution), the time-dependent laterally uniform section CD, and a fully dynamic shock BC joining the two simpler shocks. The curve BC is governed by the shock condition

$$
\frac{\partial z}{\partial t}+u(z) \frac{\partial z}{\partial x}=\frac{1}{2}\left(1+\frac{\psi-\psi_{r}}{x}\right) .
$$

Using the method of characteristics to solve (5.23) yields two subsidiary equations

$$
\frac{d x}{d t}=u(z), \quad \frac{d \psi}{d x}=\frac{1}{2}\left(1+\frac{\psi-\psi_{r}}{x}\right)
$$

The second of these can be integrated to give

$$
\psi=\psi_{r}+x+\lambda_{1} \sqrt{x}
$$

Using the linear velocity profile (4.14) and the inverse mapping (4.16), the first equation reduces to

$$
\frac{d x}{d t}=\sqrt{\alpha^{2}+4(1-\alpha) \psi}
$$

which can be integrated by substituting for $\psi$ from (5.25), making the substitution $\xi=\sqrt{x}$ to reduce the integral to standard form and hence obtain

$$
t=I_{1}\left(x, \lambda_{1}\right)+\mu_{1}
$$

where

$$
I_{1}\left(x, \lambda_{1}\right)=\frac{2}{b} \sqrt{a+b x+c \sqrt{x}}-\frac{c}{b^{3 / 2}} \ln \left(\frac{c+2 b \sqrt{x}}{2 \sqrt{b}}+\sqrt{a+b x+c \sqrt{x}}\right),
$$

with $a=\alpha^{2}+4(1-\alpha) \psi_{r}, b=4(1-\alpha)$ and $c=4(1-\alpha) \lambda_{1}$. These characteristic curves are sketched in Fig.5 using dashed arrowed lines; they emanate from the 
dot-dash curve AC, which is defined by the trajectory of the intersection between the fan-interface $\mathrm{CF}$ and the laterally uniform shock CD. The location of points on $\mathrm{AC}$ is thus given by

$$
x_{b}=\alpha t+(1-\alpha)\left(2 z_{r}+t-2 \sqrt{z_{r} t}\right) t, \quad z_{b}=z_{r}+t-2 \sqrt{z_{r} t}, \quad t_{\text {bottom }}<t<t_{3} .
$$

Each characteristic is initiated at some time $t_{o}$ from $\left(x_{b}\left(t_{o}\right), z_{b}\left(t_{o}\right)\right)$, which is shown by the grey markers on the dot-dash section of AC. On this characteristic the initial conditions imply that the constants in (5.25), (5.27) are given by

$$
\lambda_{1}\left(t_{o}\right)=\frac{\psi_{b}\left(t_{o}\right)-\psi_{r}-x_{b}\left(t_{o}\right)}{\sqrt{x_{b}\left(t_{o}\right)}}, \quad \mu_{1}\left(t_{o}\right)=t_{o}-I_{1}\left(x_{b}\left(t_{o}\right), \lambda_{1}\left(t_{o}\right)\right) .
$$

The fully dynamic part of the shock BC is therefore constructed by parameterizing the characteristics that are initiated at time $t_{o} \in\left[t_{\text {bottom }}, t\right]$ and working out their current position at time $t$. Equation (5.27) and the constants (5.30) imply that at any given time $t$ the downslope position $x_{1}\left(t, t_{o}\right)$ of the bottom shock that was initiated at time $t_{o}$, is given by solving for $x_{1} \in\left[x_{b}\left(t_{o}\right), x_{b}(t)\right]$ from the equation

$$
t=t_{o}+I_{1}\left(x_{1}, \lambda_{1}\left(t_{o}\right)\right)-I_{1}\left(x_{b}\left(t_{o}\right), \lambda_{1}\left(t_{o}\right)\right) .
$$

The corresponding height $z_{1}\left(t, t_{o}\right)$ of the shock is then given implicitly by

$$
\psi_{1}\left(x_{1}, \lambda_{1}\left(t_{o}\right)\right)=\psi_{r}+x_{1}+\lambda_{1}\left(t_{o}\right) \sqrt{x_{1}} .
$$

While we cannot write explicit formulae for $\left(x_{1}, z_{1}\right)\left(t, t_{o}\right)$, the curve BC is easily generated numerically from the sequence of equations (5.28) to (5.32). The results showing the development of the steady-state, fully dynamic and time-dependent laterally uniform sections of the lower shock appear in the righthand panels of Fig. 4 for $\alpha=1 / 2$ and $z_{r}=0.4$.

In simple shear the behaviour of the characteristic emanating from point A at $\left(x_{\text {bottom }}, 0\right)$ is particularly interesting. As $\alpha \longrightarrow 0$, the mapped discontinuity height $\psi_{r} \longrightarrow z_{r}^{2}$ and $x_{b}\left(t_{\text {bottom }}\right) \longrightarrow z_{r}^{2}$, which imply that

$$
c+2 b \sqrt{x_{b}\left(t_{\text {bottom }}\right)} \longrightarrow 0, \quad a+b x_{b}\left(t_{\text {bottom }}\right)+c \sqrt{x_{b}\left(t_{\text {bottom }}\right)} \longrightarrow 0,
$$

in (5.28). Since $\lambda_{1}\left(x_{b}\left(t_{\text {bottom }}\right)\right)=-2 z_{r}$ the constant $c$ in (5.28) is negative and

$$
I_{1}\left(x_{b}\left(t_{\text {bottom }}\right), \lambda_{1}\left(t_{\text {bottom }}\right)\right) \longrightarrow-\infty .
$$

It follows that (5.31) has a logarithmic singularity as $\alpha \longrightarrow 0$, and, therefore, point $\mathrm{B}$ can not move away from its initial position in simple-shear. As a consequence, it is not possible to generate a steady-state section of the lower shock, when $\alpha=0$, which is a rather surprising result.

\section{(e) The fully dynamic top shock}

The structure of the top shock is slightly different to that of the bottom shock, as suggested in Fig.5. There is a steady-state section EF, a fully dynamic curve FG and a laterally uniform time-dependent section GH, but this time the contact 
fan-interface intersects the steady-state shock at $\mathrm{F}$ rather than along the laterally uniform part.

The fully dynamic curve FG is governed by the shock condition

$$
\frac{\partial z}{\partial t}+u(z) \frac{\partial z}{\partial x}=\frac{1}{2}\left(\frac{z-z_{r}}{t}-1\right)
$$

since $\phi=1$ above the shock, and $\phi$ is given by (5.14) below. The method of characteristics yields two subsidiary equations

$$
\frac{d x}{d t}=u(z), \quad \frac{d z}{d t}=\frac{1}{2}\left(\frac{z-z_{r}}{t}-1\right)
$$

which are easily solved to show that the characteristic curves are described by

$$
z=z_{r}-t+\lambda_{2} \sqrt{t}, \quad x=I_{2}\left(t, \lambda_{2}\right)+\mu_{2}
$$

where

$$
I_{2}\left(t, \lambda_{2}\right)=\alpha t+2(1-\alpha) z_{r} t-(1-\alpha) t^{2}+\frac{4}{3} \lambda_{2} t^{\frac{3}{2}}
$$

The shocks are initiated along the trajectory of point $F$, which moves along the steady-state section of the upper shock EF as shown in Fig. 5. At any given time $t$ the position of $\mathrm{F}$ is found by solving the simultaneous equations (5.10), (5.22). This is achieved in practice by solving

$$
t=\frac{x_{s}}{\alpha+(1-\alpha)\left(z\left(\psi_{2}\left(x_{s}\right)\right)+z_{r}\right)},
$$

for $x_{s}$, where the function $z=z(\psi)$ is given by (4.16) and $\psi_{2}$ is the steady-state solution (5.10). The expanding fan of characteristic curves emanating from EF can then be parameterized by $x_{o} \in\left[x_{t o p}, x_{s}(t)\right]$. For a given characteristic $x_{o}$ the initial height and time of creation are

$$
z_{o}=z\left(\psi_{2}\left(x_{o}\right)\right), \quad t_{o}=\frac{x_{o}}{\alpha+(1-\alpha)\left(z_{o}+z_{r}\right)},
$$

which imply that the constants in (5.37) are given by

$$
\lambda_{2}\left(x_{o}\right)=\left(z_{o}-z_{r}+t_{o}\right) / \sqrt{t_{o}}, \quad \mu_{2}\left(x_{o}\right)=x_{o}-I_{2}\left(t_{o}, \lambda_{2}\left(x_{o}\right)\right) .
$$

The current position of the fully dynamic part of the top shock is therefore

$$
\left.\begin{array}{rl}
z_{2} & =z_{r}-t+\lambda_{2}\left(x_{o}\right) \sqrt{t} \\
x_{2} & =x_{o}+I_{2}\left(t, \lambda_{2}\left(x_{o}\right)\right)-I_{2}\left(t_{o}, \lambda_{2}\left(x_{o}\right)\right)
\end{array}\right\} x_{\text {top }}<x_{o}<x_{s}(t), t_{\text {top }}<t<t_{3} .
$$

The evolution of the three sections of the top shock are illustrated in the righthand panels of Fig. 4 for $\alpha=1 / 2$ and $z_{r}=0.4$.

\section{(f) Interaction of the dynamic top shock and the time-dependent bottom shock}

At time $t_{3}$ the upper and lower time-dependent laterally uniform shocks meet to form a third laterally uniform shock $z_{3}$ given by (5.17) over their common region 


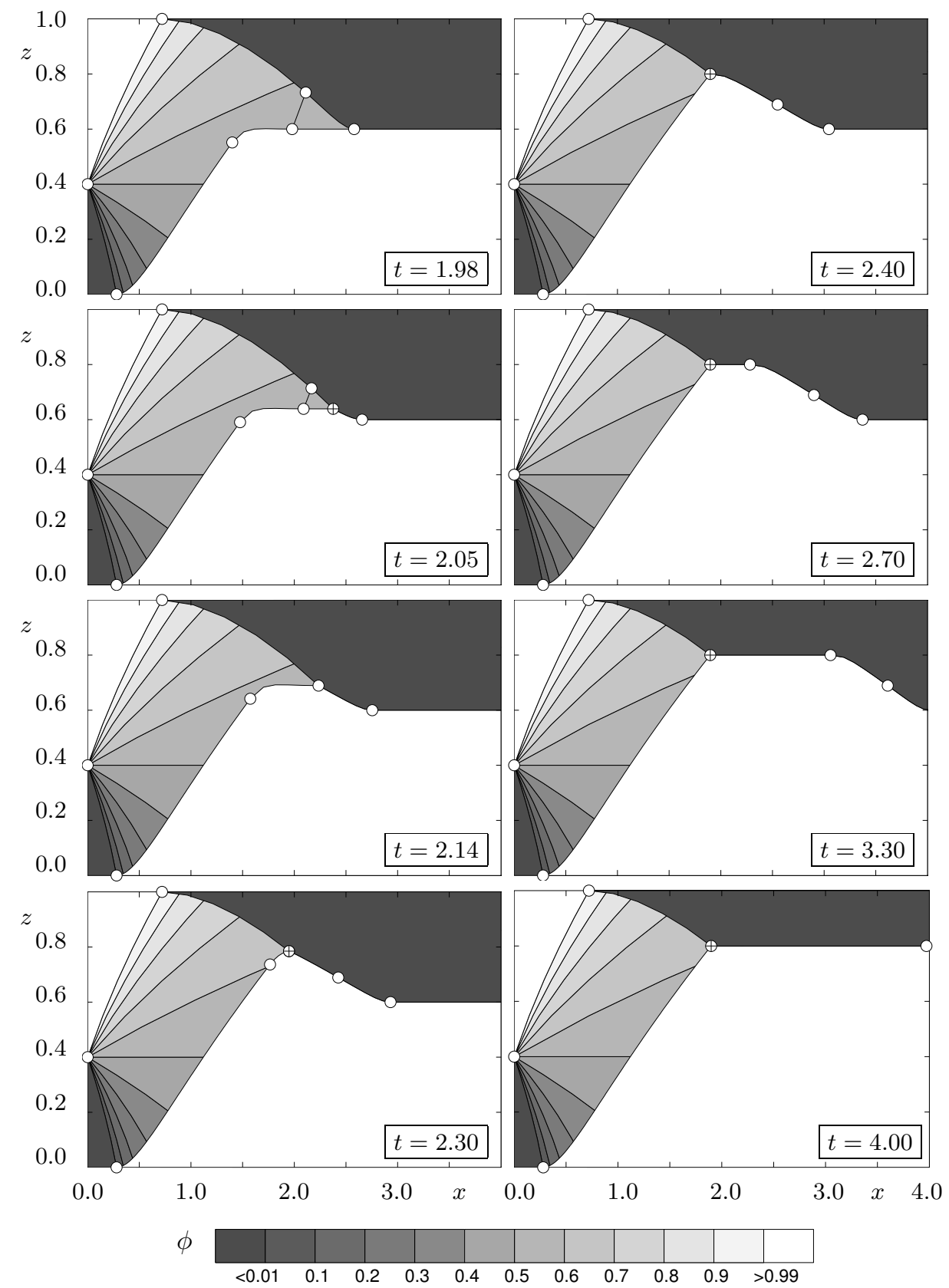

Figure 6. A series of contour plots showing the evolution of the volume fraction of small particles $\phi$ as a function of the downslope coordinate $x$ and avalanche depth $z$ for the same case as in Fig. 4 at later times. The bulk linear shear flow is from left to right and is given by parameter $\alpha=1 / 2$. The normally graded particles enter the chute at $x=0$ and have a concentration discontinuity at $z_{r}=0.4$. The white circular markers correspond to the position of the transition points between steady, fully dynamic and unsteady laterally uniform behaviour and the marker with the plus sign is the triple-point. 
of intersection. The top-left panel of Fig.6, continuing on from Fig.4, shows the solution for $\alpha=1 / 2$ and $z_{r}=0.4$. The position of the triple-point between the three shocks (shown with a ' $\oplus$ ' in Fig.6) can be found by iterating to find the characteristic $x_{\text {otriple }} \in\left[x_{t o p}, x_{s}(t)\right]$ whose corresponding shock position, given by (5.42), has the same height as the bottom shock (5.15) using

$$
\lambda_{2}\left(x_{\text {otriple }}\right)-2 \sqrt{t}+2 \sqrt{z_{r}}=0,
$$

and then substituting the result back into (5.42). The fully dynamic part of the upper shock generated by characteristics $x_{o} \in\left[x_{\text {otriple }}, x_{s}(t)\right]$ continues to evolve as before. However, the section generated by $x_{o} \in\left[x_{t o p}, x_{o t r i p l e}\right]$ has already intersected with the bottom shock at time

$$
t_{o 2}=\left(\lambda_{2}\left(x_{o}\right) / 2+\sqrt{z_{r}}\right)^{2}
$$

and at position

$$
\begin{aligned}
& z_{o 2}=z_{r}-t_{o 2}+\lambda_{2}\left(x_{o}\right) \sqrt{t_{o 2}} \\
& x_{o 2}=x_{o}+I_{2}\left(t_{o 2}, \lambda_{2}\left(x_{o}\right)\right)-I_{2}\left(t_{o}, \lambda_{2}\left(x_{o}\right)\right) .
\end{aligned}
$$

At these points, the shock is a jump from $\phi=0$ to $\phi=1$, and consequently now form part of the third shock $z_{3}$ which is governed by the conservation law (4.20), and has the simple solution

$$
x_{3}=x_{o 2}+u\left(z_{o 2}\right)\left(t-t_{o 2}\right), \quad z_{3}=z_{o 2} .
$$

As can be seen from the first three lefthand panels of Fig. 6 the triple-point moves upstream from its initial position as it generates the non-uniform section of the third shock, which is swept downstream.

\section{(g) Destruction of the fan-interface}

At $t=t_{f a n}$ the steady-upper and dynamic-lower shocks come together for the first time, eliminating the time-dependent fan (5.14), destroying the fan-interface (5.22) and ceasing the interaction between the unsteady-uniform bottom shock and the top-dynamic shock. The fan-interface destruction time $t_{f a n}$, therefore, marks a fundamental switch in behaviour of the solution. It is solved for by an iterative procedure. For an initial guess for $t_{f a n} \in\left[t_{3},\left(1+\sqrt{z_{r}}\right)^{2}\right]$ the position of the intersection between the top-steady shock (5.10) and the bottom unsteady laterally uniform shock (5.32) is found by calculating

$$
z_{\text {fan }}=z_{r}+t_{f a n}-2 \sqrt{z_{r} t_{f a n}}, \quad x_{f a n}=\left(\sqrt{1-\psi_{r}}+\sqrt{1-\psi\left(z_{f a n}\right)}\right)^{2},
$$

and then iterating $t_{f a n}$ until it lies on the fan-interface (5.22) and satisfies

$$
x_{f a n}=\left[\alpha+(1-\alpha)\left(z_{f a n}+z_{r}\right)\right] t_{f a n} .
$$

The upper bound on the interval for $t_{f a n}$ follows from the condition that the timedependent laterally uniform bottom shock must intersect with the steady top shock 


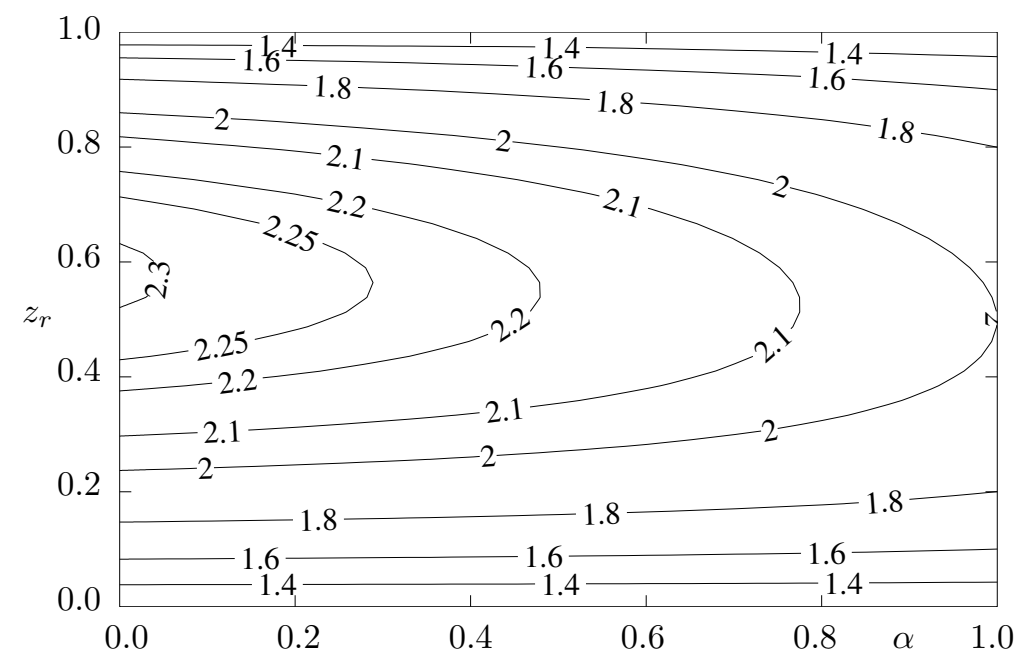

Figure 7. A contour plot of the fan-interface destruction time $t_{f a n}$ as a function of velocity profile $\alpha$ and the inflow discontinuity height $z_{r}$.

before $z=1$. The shock configuration at $t_{\text {fan }}=2.14$ is shown in Fig. 6 and the complete parameter dependence of the solution is plotted in Fig. 7. This shows that for fixed $\alpha$, the fan-destruction time has a local maximum close to $z_{r}=1 / 2$, whilst for fixed $z_{r}$ the time $t_{f a n}$ increases with increasing shear. In plug-flow $(\alpha=1)$ the fan-destruction time $t_{f a n}=t_{3}$, which is the same time that the upper and lower laterally uniform shocks initially meet.

(h) Interaction of the dynamic bottom shock and the steady top shock

For the vast majority of parameter values the dynamic bottom shock and the steady-top shock first intersect for $t>t_{\text {fan }}$ to create another section of the third shock $z_{3}$. The resulting triple-point is found iteratively by searching for the characteristic $t_{\text {otriple }} \in\left[t_{\text {bottom }}, t_{\text {contact }}\right]$, whose current bottom shock position $x_{1}\left(t, t_{\text {otriple }}\right)$ intersects with the steady-top shock (5.10), i.e. when

$$
x_{1}\left(t, t_{\text {otriple }}\right)=\left(\sqrt{1-\psi_{r}}-\lambda_{1}\left(t_{\text {otriple }}\right) / 2\right)^{2} .
$$

The triple-point coordinates are then given by (5.31) and (5.32). The fully dynamic part of the bottom shock generated by the characteristics $t_{o} \in\left[t_{\text {bottom }}, t_{\text {otriple }}\right]$ continues to evolve as before. But, the part generated by the characteristics $t_{o} \in$ $\left[t_{\text {otriple }}, t_{f a n}\right]$ intersected with the steady-top shock at point

$$
x_{o 1}=\left(\sqrt{1-\psi_{r}}-\lambda_{1}\left(t_{o}\right) / 2\right)^{2}, \quad \psi_{o 1}=\psi_{r}-x_{o 1}+2 \sqrt{\left(1-\psi_{r}\right) x_{o 1}},
$$

at time

$$
t_{o 1}=t_{o}+I_{1}\left(x_{o 1}, \lambda_{1}\left(t_{o}\right)\right)-I_{1}\left(x_{b}\left(t_{o}\right), \lambda_{1}\left(t_{o}\right)\right) .
$$

These points now generate a new section of $z_{3}$. This is governed by the inviscid Burgers equation (4.20) and has the simple solution

$$
x_{3}=x_{o 1}+u\left(z_{o 1}\right)\left(t-t_{o 1}\right), \quad z_{3}=z_{o 1},
$$




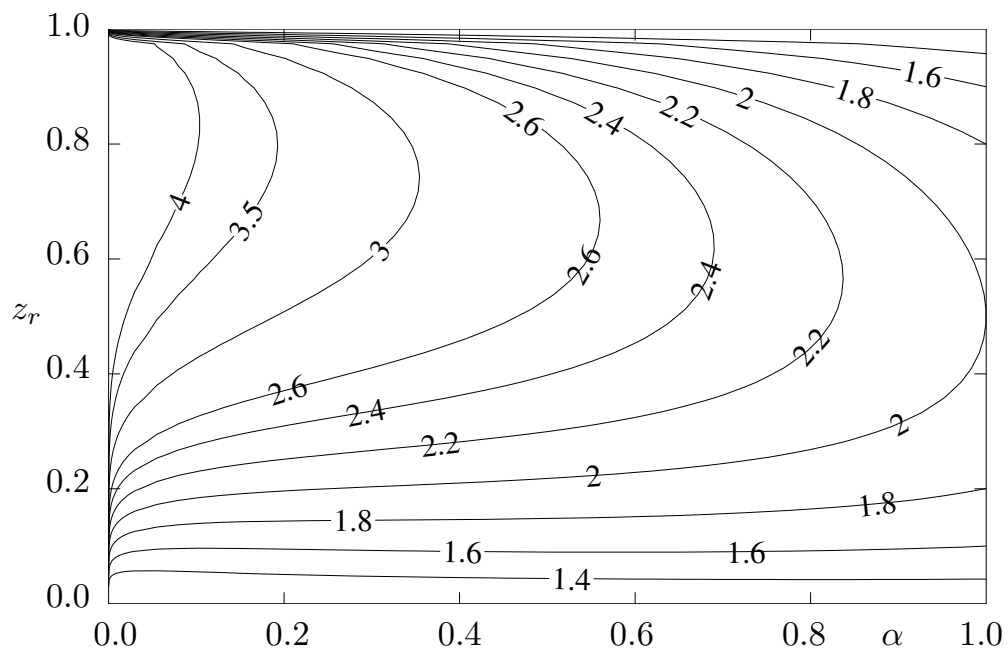

Figure 8. A contour plot of the time $t_{\text {steady }}$ for the steady-state fan fully develop as a function of velocity profile $\alpha$ and the inflow discontinuity height $z_{r}$.

where $z_{o 1}=z\left(\psi_{o 1}\right)$. The final point of this unsteady third shock is generated as point B reaches the steady-state triple-point $(5.11)$ at $\left(x_{\text {steady }}, \psi_{\text {steady }}\right)$. This point lies on the characteristic with label $t_{\text {bottom }}$ and (5.31) implies that it reaches $x_{\text {steady }}$ at time

$$
t_{\text {steady }}=t_{\text {bottom }}+I_{1}\left(x_{\text {steady }}, \lambda_{1}\left(t_{\text {bottom }}\right)\right)-I_{1}\left(x_{b}\left(t_{\text {bottom }}\right), \lambda_{1}\left(t_{\text {bottom }}\right)\right) .
$$

This time is important because it marks the point at which the steady-state concentration fan becomes fully developed. The parameter dependence of $t_{\text {steady }}$ on $\alpha$ and $z_{r}$ is illustrated in Fig. 8. In simple shear point B does not move from its initial position because of the logarithmic singularity (5.34). As a result the steadystate fan does not develop and there is a logarithmic singularity in $t_{\text {steady }}$ along the $\alpha=0$. For plug-flow, the steady-state fan becomes fully developed at the same time the upper and lower laterally uniform shocks meet, i.e. $t_{\text {steady }}=t_{3}$ when $\alpha=1$. Between these two extremes there is a maximum in $t_{\text {steady }}$, for each $\alpha$, that becomes progressively skewed to higher discontinuity heights $z_{r}$ as $\alpha$ decreases.

As we warned at the beginning of section, the bottom dynamic shock does not always intersect with the top shock after $t_{f a n}$. For a small range of parameter values in the bottom lefthand corner of Fig. 8, i.e. for $\alpha$ and $z_{r}$ small, the dynamic bottom shock develops a pronounced peak as shown in Fig. 9. This can intersect with the steady top shock prior to $t_{\text {fan }}$, dividing the fan in two, and changing the topology of the problem. Numerical simulations of a similar-cut off phenomenon by Thornton, Gray \& Hogg (2005) suggest a detached section of $z_{3}$ forms, which expands and merges with the rest of $z_{3}$ as the enclosed part of the fan is destroyed.

For the majority of parameter values, however, a laterally uniform steady-state shock $\psi_{3}$, that was defined in (5.12), is generated for $t>t_{\text {steady }}$. The full structure and development of $z_{3}$ is illustrated in the remaining panels of Fig. 6 . It consists of four sections. A laterally uniform region at height $1-z_{r}$ generated by the initial data, a section formed from the interaction of the dynamic top shock and the laterally uniform bottom shock (5.47), a section generated by the interaction of the 


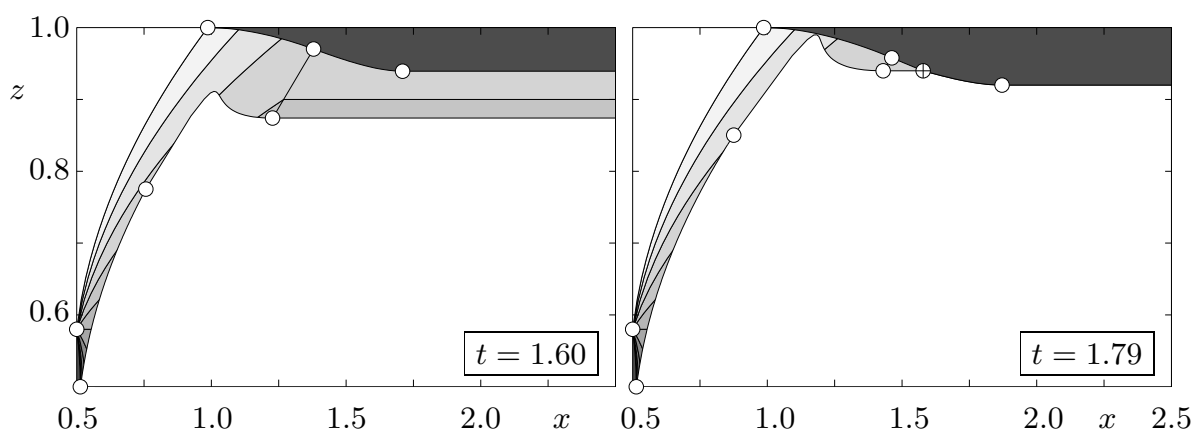

Figure 9. Two contour plots showing a zoomed in view of the small particle concentration for $\alpha=0.01$ and $z_{r}=0.16$. At high shear and low $z_{r}$ the dynamic bottom shock develops a peak (left) that intersects (right) with the top steady shock before $t=t_{\text {fan }}$. Note, the vertical and horizontal scales have changed.

steady-state top shock and the dynamic bottom shock (5.53), and, a steady-state region at height $1-\psi_{r}$. The markers in Fig. 6 indicate the transitions between these regions. As in the first problem in $\S 4$ the fully dynamic parts of the third shock $z_{3}$ steepen and break in finite time $t_{\text {break }}$, which may be either before or after $t_{\text {steady }}$. Although the subsequent breaking is complex, this region is swept downstream, and locally a steady-state is rapidly attained provided the logarithmic singularity in simple-shear is avoided. This is illustrated in the last panel of Fig. 6, where the local solution in the domain of interest has reached steady-state at $t=4$. In this fully developed region, large particles enter from the lower left and move up through the expansion fan, before separating out, whilst the small particles enter from the upper left move down through the fan and separate into a pure phase. In this way an inversely graded layer is formed, which is the basic building block for many pattern formation processes.

\section{Discussion and conclusions}

In this paper two exact solutions to the segregation theory of Gray \& Thornton (2005) have been derived for steady uniform chute flows. These graphically illustrate some of the key physical effects that develop during the segregation process, including:- concentration shocks, concentration expansions, non-centred expanding shock regions, the creation of inversely-graded layers and the steepening and ultimate breaking of monotonely decreasing sections of the shock between these layers. The non-dimensional segregation number $S_{r}$ determines the strength of the segregation and may be calibrated from experiment. In steady-uniform flows a spatial and temporal stretching transformation (3.2) can be used to reduce the segregation equation to a convenient parameterless form. The resulting solutions are dependent only on the assumed velocity profile with depth and the boundary and initial conditions. By constructing exact solutions we have, therefore, been able to map out the entire parameter space for the two problems. This not only provides important test cases for numerical shock-capturing methods to solve more general problems, but, yields implicit and explicit formulae for the key times and locations at which the behaviour of the solution fundamentally changes. Of particular interest is the fact 
that in simple shear the logarithmic singularity in (5.34) prevents the formation of a steady-state lower shock. This is qualitatively different to solutions with slip at the base, where a steady-state lower shock is formed in finite time. In addition, we have also identified a region of parameter space, where the non-linearity leads to the pinching off of a section of the expansion fan, changing the topology of the solution. Regardless of the precise nature of the initial and boundary conditions, or the topology changes that occur during the flow, particles separate out into inversely-graded layers sufficiently far downstream and the complex shock breaking behaviour in monotonely decreasing regions is advected downstream.

\section{Acknowledgements}

The authors gratefully acknowledge the generous support of the Isaac Newton Institute Programme on "Granular and particle-laden flows", which was held in Cambridge from September-December 2003. Nico Gray was also supported by an EPSRC Advanced Research Fellowship (GR/S50052/01 \& GR/50069/01) and Anthony Thornton by an EPSRC Doctoral Training Account. Michael Shearer was supported in part by Grant DMS-0244491 from the National Science Foundation.

\section{References}

Bridgwater, J. 1976. Fundamental powder mixing mechanisms. Powder Tech. 15, 215-236.

Chadwick, P., 1976. Continuum Mechanics. Concise theory and problems. George Allen \& Unwin (republished Dover 1999).

Davidson J.F., Scott D.M., Bird P.A, Herbert O., Powell A.A. \& Ramsey H.V.M. 2000. Granular motion in a rotary kiln: the transition from avalanching to rolling. KONA powder and particle 18, 149-155.

Dolgunin, V.N. \& Ukolov A.A. 1995. Segregation modelling of particle rapid gravity flow. Powder Tech. 83, 95-103.

Eglit M.E. 1983. Some mathematical models of snow avalanches. In Advances in mechanics and the flow of granular materials (ed. M. Shahinpoor), Clausthal-Zellerfeld and Gulf Publishing Company 2, 577-588.

Gray, J.M.N.T. \& Chugunov, V.A. 2005. Particle-size segregation and turbulent remixing. in prep

Gray, J.M.N.T. \& Hutter K. 1997. Pattern formation in granular avalanches. Contin. Mech. Thermodyn. 9(6), 341-345.

Gray, J.M.N.T., Tai, Y.-C. \& Noelle, S. 2003. Shock waves, dead-zones and particle-free regions in rapid granular free surface flows. J. Fluid. Mech. 491, 161-181.

Gray, J.M.N.T. \& Thornton A.R. 2005. A theory for particle size segregation in shallow granular free-surface flows. Proc. Roy. Soc A in press.

Gray, J.M.N.T., Wieland, M. \& Hutter K. 1999. Free surface flow of cohesionless granular avalanches over complex basal topography. Proc. Roy. Soc. A 455, 1841-1874.

Grigorian, S.S., Eglit, M.E. \& Iakimov, Iu.L. 1967. New statement and solution of the problem of the motion of snow avalanche. In Snow, Avalanches 8 Glaciers. Tr. Vysokogornogo Geofizich Inst. 12.

Iverson, R.M. 1997. The physics of debris flows. Reviews of Geophysics 35(3), 245-296.

Iverson, R.M. \& J.W. Vallance 2001. New views of granular mass flows. Geology 29(2), $115-118$. 
Middleton, G.V. \& Hampton, M.A. 1976. Subaqueous sediment transport and deposition by sediment gravity flows. In Marine sediment transport and environmental management (ed. D.J. Stanley \& D.J.P. Swift), Wiley, 197-218.

Pouliquen, O. Delour, J. \& Savage, S.B. 1997. Fingering in granular flows. Nature, 386, 816-817.

Savage, S. B. and Hutter, K. 1989. The motion of a finite mass of granular material down a rough incline. J. Fluid Mech. 199, 177-215.

Savage S.B \& Lun, C.K.K. 1988. Particle size segregation in inclined chute flow of dry cohesionless granular solids. J. Fluid. Mech. 189, 311-335.

Sethian, J.A. 1999. Level set methods and fast marching methods Cambridge University Press 378pp.

Shinbrot, T. \& Muzzio F.J. 2000. Nonequilibrium patterns in granular mixing and segregation. Physics Today, March 2000, 25-30.

Thomas, N. 2000. Reverse and intermediate segregation of large beads in dry granular media. Phys. Rev. E.69(1), 961-974.

Thornton, A.R., Gray, J.M.N.T. \& Hogg, A.J. 2005 A three-phase mixture theory for particle size segregation in shallow granular free-surface flows. J. Fluid Mech. Submitted.

Vallance, J.W. 2000. Lahars. Encyclopedia of Volcanoes, pp. 601-616, Academic press.

Vallance, J.W. \& Savage S.B. 2000. Particle segregation in granular flows down chutes. In IUTAM Symposium on segregation in granular materials (Eds. A.D. Rosato \& D.L. Blackmore), Kluwer, 31-51.

Williams, J.C. 1968. The mixing of dry powders. Powder Tech. 2, 13-20.

Wills B.A. 1979. Mineral processing technology. Pergamon. 